\title{
Methods for multi-detector burst gravitational wave search
}

\author{
I S Heng ${ }^{1}$, F Salemi ${ }^{2}$ and A Ortolan ${ }^{3}$ \\ ${ }^{1}$ Max-Planck-Institut fuer Gravitationsphysik, Albert-Einstein-Institut, Aussenstelle Hannover, \\ Callinstrasse 38, D-30167 Hannover, Germany \\ ${ }^{2}$ INFN and Dipartimento di Fisica, Università di Ferrara, Via Paradiso 12, I-44100 Ferrara, Italy \\ ${ }^{3}$ Laboratori Nazionali di Legnaro, Istituto Nazionale di Fisica Nucleare, Viale dell'Università 2, \\ I-35020 Legnaro, Padova, Italy \\ E-mail: siong@aei.mpg.de, francesco.salemi@fe.infn.it and antonello.ortolan@lnl.infn.it
}

Received 24 April 2003

Published 7 August 2003

Online at stacks.iop.org/CQG/20/S617

\begin{abstract}
In this paper, a review of methods of data exchange and multi-detector analysis is given. Details of data exchange parameters will also be discussed. We focus on the coherent network search which turns out to be a fundamental step towards the overcoming of ambiguity in parameter estimation of a gravitational wave in the low signal-to-noise ratio regime.
\end{abstract}

PACS numbers: $07.05 . \mathrm{Kf}, 95.85 . \mathrm{Sz}$

\section{Introduction}

In the search for burst gravitational waves, the need to distinguish genuine signals, which are observable almost instantaneously in widely-spaced detectors ${ }^{4}$, from spurious transients is crucial. Ideally, one would apply an optimal filter built for the desired waveform on the output from a single detector and apply the $\chi^{2}$-test to the filtered output. However, given current astrophysical predictions and gravitational wave detector sensitivities, one expects the signal-to-noise ratios (SNRs) and rates of burst gravitational waves to be very low. At low SNRs (for instance, SNR $<15$ for the AURIGA first run), there is a large ambiguity in the estimation of the burst parameters, namely arrival time and amplitude [1]. This uncertainty makes genuine burst gravitational wave signals almost indistinguishable from events arising from unmodelled environmental noise sources. In fact, the $\chi^{2}$-test does not have enough statistical power to identify the signal template and/or parameters in the low SNR regime, as previously shown for resonant-mass detectors by Baggio et al [2].

4 Within the light travel time through the Earth, approximately $43 \mathrm{~ms}$. 
Thus, to make the distinction between signal and noise, one must cross-correlate data from multiple detectors to reduce contributions from spurious environmental noise occurring around the individual detectors and increase the SNR of gravitational wave bursts. Hence, methods for performing multi-detector burst searches and the choice of data exchange parameters are a crucial part of the burst gravitational wave search pipeline.

For any multi-detector search, information about the statistics of the noise, such as its variance and possibly even higher-order moments of its distribution, must be exchanged in addition to the parameters of signal candidates. A correct representation of the noise in the exchanged data allows for the accurate determination (by means of a Monte Carlo simulation) of the level of false alarms and false dismissals for the network. Moreover, the search method should also be able to handle the output from different burst analysis pipelines.

Burst gravitational wave searches on data from multiple, widely-spaced detectors have previously been performed [3, 4] to put upper limits on the rates and amplitudes of burst gravitational waves impinging on the earth. The International Gravitational Event Collaboration (IGEC) developed a framework (data format and analysis tools) within which a thorough analysis of the triggers from the burst search pipeline of five resonant-mass detectors was performed [5, 6]. Though this framework allows for a statistically robust analysis, which has given the best upper limits on gravitational wave events in our galaxy, it is currently restricted to the analysis of triggers from resonant-mass detectors.

A network of interferometric and resonant-mass detectors is desirable because having more detectors in a network reduces the false alarm rate. Resonant-mass detectors have comparable sensitivities with interferometers within a bandwidth of $50-80 \mathrm{~Hz}$ around $1 \mathrm{kHz}$. Some burst gravitational wave signals such as those from black-hole ringdowns have a detection bandwidth narrower than that of the resonant-mass detectors. For the detection of such signals, only the minimum value of the strain noise curve is relevant; in this case, interferometric and resonant-mass detectors are comparable. On top of this, resonant-mass detectors have excellent long term duty cycle and would provide a substantial improvement to sky coverage [6]. An interferometric and resonant-mass detector network can also test the spin- 2 nature of gravitational waves (absence of scalar spin-0 graviton) while a network of interferometers is, by construction, completely insensitive to spin- 0 gravitational waves.

Astone and Schutz have previously discussed the idea of narrowbanding interferometers for the purpose of performing a burst search between interferometers and resonant-mass detectors [7]. The drawback to this method is that narrowbanding reduces the sensitivity of the interferometers to burst signals, but it could be useful for those astrophysical templates (e.g., quasi-normal ringdown of perturbed black holes) whose spectral power is concentrated within a narrow band.

In this paper, we describe three methods for performing burst searches between interferometers and resonant-mass detectors. The first method is a coincidence search and it amounts to an extension of the IGEC framework to include the parameters from interferometer burst search algorithms (also known as event trigger generators or ETGs). The second method is the externally triggered search method previously proposed by Finn et al [8]. The third method is what we call a 'coherent network search'. We will give an outline of each method and discuss the data parameters one would have to exchange. It should be noted that all three search methods take advantage of the fact that burst signals, by definition, last no more than a few milliseconds and, therefore, we only need to exchange a few seconds of data around the occurrence of a trigger. This reduces the size of the exchanged data and also the computational power required for the analysis. 


\section{Search methods}

\subsection{Coincident trigger search}

The most straightforward approach to a burst gravitational wave search is to search for coincident excitations between widely-spaced detectors. For such an analysis, candidate burst event lists are independently generated for each detector by applying a threshold to extract large SNR excitations. A search for coincident arrival times is then performed. In addition to correlating the event arrival times, one may also apply additional constraints to other signal parameters such as its amplitude or the orientation of the detector with respect to a fixed direction in the sky (for non-isotropic source distribution, e.g. galactic sources).

This kind of analysis has been used in several searches in the past and, most recently, by the IGEC $[5,6]$. The IGEC have established a completed framework for exchanging data in view of performing a coincidence search. Within this framework, it is mandatory to exchange the time of occurrence, amplitude and duration of a trigger and the time spans of detector operation. In addition, one has to exchange the variance of the noise distribution as well as its skewness and kurtosis (third and fourth moments).

For a burst gravitational wave search between triggers from resonant-mass and interferometric detectors, we would extend this framework to include parameters from interferometer ETGs by exchanging information about the frequency band over which an event stretches.

By using the higher-order moments, Bienayme's inequality allows an estimate of the false alarm probability and detection efficiency of each event, as a function of the corresponding SNR. Although Bienamye's inequality is a non-parametric test which gives a very conservative estimate of the false alarm probabilities, it provides more statistical robustness in the presence of non-stationary and/or non-Gaussian noise.

However, if we want to perform such a trigger-based coincidence search between resonantmass and interferometric gravitational wave detectors, we have to fully characterize the different search algorithms so that we can exchange homogeneous definitions of signal parameters such as amplitude and SNR. To this end, one needs to perform Monte Carlo simulations where the effect of each burst search pipeline on particular signal templates is studied. Such simulations and software signal injections in the detector noise are currently being performed by the LIGO burst analysis group and the AURIGA group.

Another approach to this problem would be to apply a new burst search algorithm that restricts interferometer search to the resonant-mass detector's band and apply the optimal filter for the resonant-mass detector. This way, we ensure that the same quantities are compared when searching for coincidences. The required filter should express the amplitude of an event observed by an interferometer in terms of the one observed by a resonant-mass detector. However, such techniques are not straightforward and beyond the scope of this paper.

\subsection{Externally triggered search}

Another approach to multi-detector burst searches would be to use a detector with fewer triggers (such as gamma ray bursts and resonant-mass detectors) to trigger the cross-correlation of data from detectors with a high trigger rate (such as the current interferometeric detectors). This is the externally triggered search laid out in Finn et al [8].

For this search, the cross-correlation statistic of the output of two gw detectors is calculated for a time window $T$ when a trigger is observed. That is, one calculates 


$$
X=\int_{0}^{T} \int_{0}^{T} \mathrm{~d} t \mathrm{~d} t^{\prime} x_{1}\left(t_{1, \gamma}-t\right) Q\left(\left|t-t^{\prime}\right|\right) x_{2}\left(t_{2, \gamma}-t^{\prime}\right),
$$

where $T$ is the time window around a bar trigger within which a trigger is expected in the interferometer and $Q$ is the filter kernel, details of which are discussed in [8]; here, $x_{I}$ and $t_{I, \gamma}$ are the data samples and the arrival time of the bar trigger for the Ith detector, respectively. The same statistic is also calculated for the same period of time when no bar triggers are observed. A statistically significant difference between on- and off-source correlations supports the presence of a signal. Finn et al suggested the use of Student's $t$-test to statistically test for the difference between the two distributions. However, for robustness, Tricarico et al [9] recommended the use of the Mann-Whitney $u$-test, which is useful for systems plagued by unmodelled noise backgrounds and/or quasi-stationary noises; the $u$-test only demands that the shapes of the two distributions (off and on populations) are identical and that the two statistical samples are independently drawn. If the requirements of the Mann-Whitney test are met, then the one-tailed $z$-test can be applied to its outcome $u$.

\subsection{Coherent network search}

Finally, we discuss the coherent network search method. With this method, the outputs from all the detectors in the network are linearly and coherently combined using their phase information. For instance, by maximizing a network likelihood for the presence of a specified signal in the different data streams. The method can be seen as a vector matched filter. If the noise from the individual detectors of the network is not correlated, then the probability of observing a specific set of data on the different detectors is simply proportional to the sum of the log-likelihood ratios of each detector. In the coherent analysis, it should be set only at one detection threshold. Under the assumption of Gaussian noise, the coherent analysis can be made optimal, in the sense of Wiener filter theory.

We begin by calculating the log-likelihood function $\Lambda$ of the network with respect to an impulsive plane wave of amplitude $A$ impinging on the detectors, and then look for the minimum of $\Lambda$ over the gw parameters. This is equivalent to the $\chi^{2}$-statistic of the data of the detectors in the network obtained by a fit to a given plane gw burst. The network log-likelihood is given by

$$
\left\{\begin{array}{l}
\chi^{2} \equiv \Lambda\left(A, t_{0}, \hat{k}, \Psi\right)=\frac{1}{2} \sum_{I=1}^{N} \sum_{i, j}^{M_{N}} \mu_{I, i j}\left[x_{I}\left(t_{i}\right)-X_{I}\left(t_{i}\right)\right]\left[x_{I}\left(t_{j}\right)-X_{I}\left(t_{j}\right)\right] \\
X_{I}\left(t_{i}\right)=A s_{I}(\vartheta, \varphi, \Psi) f_{I}\left(t_{i}-t_{0}-\mathbf{r}_{I} \cdot \hat{\mathbf{k}} / c\right)
\end{array}\right.
$$

where $\mu_{I, i j}$ is the inverse of the cross-correlation matrix of the Ith detector noise, $x_{I}\left(t_{i}\right)$ is the dataset of length $M_{I}$ to be searched for the signal template, $\hat{\mathbf{k}}$ is the wave unit vector with angles $\vartheta$ and $\varphi, \Psi$ is the polarization angle, $\mathbf{r}_{I}$ is the position vector of the $I$ th detector in the array with respect to a geocentric coordinate system and $t_{0}$ is the signal arrival time at the centre of the Earth. Here we make explicit the dependence of the detector output $X_{I}\left(t_{i}\right)$ on the signal template $f_{I}$ and the antenna pattern $s_{I}$, defined in the standard way as

$$
s_{I}(\vartheta, \varphi, \Psi) \equiv \begin{cases}W_{k l} n_{I}^{k} n_{I}^{l} & \text { (bars) } \\ W_{k l}\left(n_{I}^{k} n_{I}^{l}-m_{I}^{k} m_{I}^{l}\right) & \text { (ifos) }\end{cases}
$$

where $W_{k l}$ is the gravitational wave polarization tensor and $\hat{\mathbf{n}}$ and $\hat{\mathbf{m}}$ are the unit vectors along the local reference frame of the Ith detector. Note that the above expressions can be easily generalized to time-dependent polarization angle introducing the two templates for the ' + ' and ' $X$ ' polarizations. For any given sky direction, $\hat{\mathbf{k}}$, arrival time, $t_{0}$, and polarization, $\Psi$, the logarithmic likelihood of the network has to be minimized. It can be shown that $\Lambda$ reaches a 
minimum when the amplitude $A$ is simply the weighted sum of the optimal estimates on the single detectors [2]

$$
\left\{\begin{array}{l}
A_{\mathrm{opt}}\left(t_{0}, \vartheta, \varphi, \Psi\right)=\sum_{I=1}^{N} \frac{A_{I}\left(t_{0}, \vartheta, \varphi, \Psi\right)}{\sigma_{I}^{2}} / \sum_{I}^{N} \frac{1}{\sigma_{I}^{2}} \\
\sigma_{I}=\left(\frac{1}{2 \pi} \int_{-\infty}^{+\infty}\left|f_{I}(\omega)\right|^{2} / S_{I}(\omega) \mathrm{d} \omega\right)^{-1 / 2},
\end{array}\right.
$$

where $\sigma_{I}$ is the standard deviation of the noise in the Ith detector and $S_{I}(\omega)$ is the spectral density of the noise. From the above equation, one can see that the weighted sum automatically selects which detector in the network to consider in the analysis because it reduces the contribution of low SNR detectors with respect to the desired signal template.

To properly determine the amplitude, arrival time and $\chi^{2}$ estimators, we have to assume that the detector noise is a linear superposition of deterministic signals (either gravitational waves or environmental events) and quasi-stationary noise. The Gaussianity of the data can be checked a priori by performing Monte Carlo simulations where signals with known waveforms are injected into the detector noise. The hypothesis testing of signal detection with the network is based on the overall $\chi^{2}$. The rejection of the null hypothesis is usually achieved by means of a threshold crossing of some signal parameter (e.g., amplitude), which corresponds to the sole (and unavoidable) nonlinear operation within the framework of the proposed search. The single threshold value will fix the global false alarm and false dismissal probabilities.

A coherent network search can be set up as follows: (i) any detector in the network can raise a trigger (detection of candidate events); (ii) all the detectors in the network should exchange a short stretch of whitened data $w_{I i}$ around the trigger, together with the adopted whitening filter; (iii) for a given template, the $\chi^{2}$-statistic of the network can then be calculated by means of equations (3) and (4)

$$
\chi^{2}=\sum_{I=1}^{N} \frac{\sum_{i}^{M_{I}} w_{I i}^{2}}{\sigma_{I w}^{2}}-\frac{\left(A_{\mathrm{opt}}\left(t_{0}, \vartheta, \varphi, \Psi\right)\right)^{2}}{\sigma_{\mathrm{opt}}^{2}},
$$

where $\sigma_{I w}$ is the standard deviation of the whitened data; (iv) once the likelihood function is calculated, two thresholds (set by signal injection studies) can be applied to the $\chi^{2}$ and $A_{\text {opt }}^{2}$ outcomes, to determine the presence or absence of a genuine gravitational wave signal.

To further test gw events against spurious excitations we can resort to the distinctive properties of the Riemann tensor (transverse and traceless) associated with a plane gw. It can be shown that there are three quantities, the eigenvalues of the polarization tensor $W_{i j}$, which are invariant under the group of spatial rotation $O(3)$ [10]. These quantities are basically the linear, quadratic and cubic combinations of the detector outputs and are random variables with distributions that can be calculated by means of a Monte Carlo which makes use of the noise distributions and orientation information of the individual detectors in the network.

\section{Summary and discussion}

We have just presented three methods for performing multi-detector burst gravitational wave searches. The search for coincidence triggers is the most straightforward method one could use to search for burst gravitational waves. The need to only exchange parameters of individual events and statistical characteristics of the noise allows for the exchange of compact data files between different detector groups. Moreover, the analysis has been widely used in the past and is well understood. However, the problem of comparing homogeneous trigger parameters between interferometers and resonant-mass detectors is quite complex and requires more investigation. 
The externally triggered search on the other hand would use triggers from resonant-mass detectors to trigger a search in two or more interferometers. It makes no assumption about the waveform of the gravitational wave signal. Moreover, because this search is comparing the statistics of a large number of cross-correlated segments, it is in effect digging into the noise of interferometric detectors.

The use of a sole threshold makes the coherent network search an extremely powerful method. A single threshold greatly increases the overall efficiency of the network. It overcomes the intrinsic limits connected with the ambiguity of parameter estimation in single detectors and allows for a stronger $\chi^{2}$-statistical analysis.

\section{References}

[1] Crivelli-Visconti V et al 1998 Phys. Rev. D 57 2045-50

[2] Baggio L et al 2000 Phys. Rev. D 61102001

[3] Astone P et al 1999 Phys. Rev. D 12122001

[4] Astone P et al 1999 Astropart. Phys. 10 83-92

[5] Allen Z A et al 2000 Phys. Rev. Lett. 85 5046-50

[6] Astone P et al 2003 Preprint astro-ph/0302482 (Phys. Rev. D to be published)

[7] Astone P, Lobo A and Schutz B 1994 Class. Quantum Grav. 11 2093-112

[8] Finn L S, Mohanty S D and Romano J D 1999 Phys. Rev. D 60121101

[9] Tricarico P et al 2001 Phys. Rev. D 63082002

[10] Cerdonio M et al 1993 Phys. Rev. Lett. 71 4107-10 